\title{
Evolution Cipher against Differential Power Attack
}

\author{
Tang ming ${ }^{1,2}$, Meng Qinshu ${ }^{2}$, Zhang Huanguo ${ }^{1,2}, \mathrm{Gao} \mathrm{Si}^{2}$, Dou $\mathrm{Qin}^{2}$, Shen Fei ${ }^{2}, \mathrm{Li} \mathrm{Du}^{2}$ \\ 1(State Key Lab. of AIS\&TC, Ministry of Education, Wuhan University in China, m.tang@126.com) \\ 2(School of Computer, Wuhan University, Wuhan 430072, China)
}

\begin{abstract}
Differntial Power Attack (DPA) is one kind of Side Channel Attacks (SCAs). There are two phases in DPA attacks: sample collection and statistical analysis, which can be utilized to construct different countermeasures against DPAs, such as balancing technologies. We propose a novel methodology to implement a secure DPA resistant crypto processor and we name this as evolutionary cipher(EVOC). EVOC can effectively resist DPA attacks, because it destroys the differential power computation proposed by Kocher. Moreover, EVOC opens up a new idea to design secure cryptographic algorithm to resist both DPA attacks and some other attacks. Designing principles of evolution cipher is useful for other dynamic cryptographic algorithms. We prove that our scheme has theoretically and practically proven security.
\end{abstract}

Keywords: evolution cipher, differential power attack, DPA resistance, dynamic countermeasure

\section{Introduction}

Security on communication, computation, and storage has been concerned for a long time.Cryptographic algorithms, including symmetric ciphers, public-key ciphers, and hash functions, form a set of primitives that can be used as building blocks to construct security mechanisms that target specific objectives ${ }^{[1]}$. Cryptosystem designers frequently assume that secrets will be manipulated in closed, reliable computing environments ${ }^{[2]}$. Based on this “ separation of concerns”, many theory analysis have been well studied, such as differential analysis ${ }^{[3]}$, linear analysis ${ }^{[4]}$.

However, in practice, even if one cryptographic algorithm has been proved to be resistant against all existing theory analyzing methods, security mechanisms based on this cryptographic algorithm are not proved to be safety or reliable. Because this kind of mechanisms may be vulnerable to side channel attacks(SCAs).

In cryptography, a side channel attack is any attack based on information gained from the physical 
implementation of a cryptosystem, rather than brute force or theoretical weaknesses in the algorithms (compare cryptanalysis) ${ }^{[5]}$.

Over the passed 10 years, there existed some effective SCA attacks such as Power attacks(combined with SPA, DPA, High-Order DPAs), Fault attacks(Spike, Glitch, Optical, Electromagnetic), Timing attacks, and Electromagnetic analysis etc.

This paper focuses on countermeasures against DPA (Differential Power Attack) which is one of the great concerning SCA attacks. DPA is a powerful tool that allows cryptanalysts to extract secret keys and compromise the security of semiconductors and tamper-resistant devices by analyzing their power consumption. Simple Power Analysis (SPA) is a simpler form of the attack that does not require statistical analysis. Unlike physical attacks, SPA and DPA attacks are non-invasive, easily-automated, and can be mounted without knowing the design of the target device.

Power analysis attacks cannot generally be detected by a device, since the adversary's monitoring is normally passive. In addition, the attack is non-invasive. As a result, physical enclosures, auditing capabilities, and attack detectors are ineffective. Instead, cryptosystem engineers must ensure that devices' power variations do not reveal information usable by adversaries. Several countermeasures were pro-posed in order to protect implementations of cryptographic al-gorithms.

These countermeasures can be classified as: masking methods, balancing, and random timing. Using randomly selected masking code to rewrite key operations, masked output data were given in masking strategy ${ }^{[6]}$. Based on DPA Leakage Models to quantitatively evaluate leakage info and design balanced circuit, balancing ${ }^{[7]}$ expect to ensure balanced or almost balanced power leakage. Random timing technology ${ }^{[8]}$ and disturbing clock frequency ${ }^{[9]}$ can increase uncertainty of certain power sample by attackers. Unfortunately, these existing countermeasures are all insuficient against DPA effectively, and we'll carefully introduce and analyze these 3 kinds of methods in section 3. This paper proposes a new method to resist DPAs which are based on the power analyzing model built by kocher ${ }^{[2]}$. Our strategy combined intelligent computing algorithm and cipher design policies to ensure both dynamic structure and high security of cryptographic algorithm. We call this strategy as evolutionary cipher, and have made some progress on automatic design SBOX ${ }^{[10,11]}$, bent 
function $^{[12]}$, hash functions ${ }^{[13]}$ with evolutionary cipher.

The reason why Evolutionary cipher can resist DPA attacks is basing on dynamic unpredictable structure which can destroy the differential power analyzing model proposed by kocher $^{[2]}$, so that can prevent attackers to identify correct data even if they have collected enough power cycles and filtered random disturbing elements. Section 4 and 5 illustrate and prove security and effectiveness of evolutionary cipher against DPA attacks respectively.

The organization of this paper is: section 2 carefully introduces DPA attacks; section 3 analyzes three different existing and widely used countermeasures against DPAs; section 4 elaborately introduces how evolutionary cipher can resist DPA attacks from designing principles, the reason of resistance against DPAs, and security proof; section 5 makes expensive experiments to verify effectiveness of evolutionary cipher against DPAs; finally last section makes conclusion and future work.

\section{Introduction of DPA}

Over the past ten years, there have been some new DPA attacks, but main idea and principles of most DPA attacks ${ }^{[14]}$ are originated from kocher ${ }^{[2]}$. Like other SCAs, DPA attacks are composed of 2 phases: one is data collection, and the other is statistical analyzing.

1) Data collection: time is the character of this phase. If power cycles are drawn in two dimension, horizontal axis and vertical axis are timing and power respectively.

2) Statistical analyzing: adversary in this step tries to find the correslation between collected power data and key in cryptography.

The following steps provide an example of a DPA attack process proposed by kocher ${ }^{[2]}$.

1) Attackers choose a key-dependent selection function D. In this case, the selection function would has the form $D\left(C_{i}, b, k_{s}\right)$, where $\mathrm{Ki}$ is some keyi information, $\mathrm{C}$ is a ciphertext and $\mathrm{b}$ is the return value of $\mathrm{D}$ function. 
2) Attackers could observe m encrypting operations and capture two kinds of infomation as following:

First: $C_{i}$ represents cipher text which is corresponding to one power trace;

Second: k samples are collection power points and each sample is related to a certain time point.

3) Statistically analyzing to get $k_{s}$ :

First: to get the value of $T_{i}[j]$ for certain power sample, $\mathrm{i}$ is the $\mathrm{i}^{\text {th }}$ power sample and $\mathrm{j}$ respects the $\mathrm{j}^{\text {th }}$ sample point;

Second: to compute the value of differential power based on function 1 , and only $C_{i}$ and $T_{i}[j]$ are variable.

$$
\begin{aligned}
& \Delta D[j]=\frac{\sum_{i=1}^{m} D\left(C_{i}, b, K_{s}\right) T_{i}[j]}{\sum_{i=1}^{m} D\left(C_{i}, b, K_{s}\right)}-\frac{\sum_{i=1}^{m}\left(1-D\left(C_{i}, b, K_{s}\right)\right) T_{i}[j]}{\sum_{i=1}^{m}\left(1-D\left(C_{i}, b, K_{s}\right)\right)} \\
& \approx 2\left(\frac{\sum_{i=1}^{m} D\left(C_{i}, b, K_{s}\right) T_{i}[j]}{\sum_{i=1}^{m} D\left(C_{i}, b, K_{s}\right)}-\frac{\sum_{i=1}^{m} T_{i}[j]}{m}\right)
\end{aligned}
$$

Third: If $k_{s}$ is incorrect, $\lim _{m \rightarrow \infty} \Delta D[j] \approx 0$; adversely if $k_{s}$ is correct, the computed value of $\Delta D[j]$ will not be zero and show spikes in regions where $\mathrm{D}$ is correlated to the values being processed. This conclusion has been proofed by kocher ${ }^{[2]}$.

While the effects of a single transistor switching would be normally be impossible to identify from direct observations of a device's power consumption, the statistical operations used in DPA are able to reliably identify extraordinarily small differences in power consumption. And we find that selection function $\mathrm{D}$ is the most important operation in data analyzing.

\section{Existing Countermeasures for DPA}

\subsection{Masking}

The masking technique is the most widely used countermeasure against power analysis and timing attacks at a software level and also the most powerful software countermeasure against side channel 
$\operatorname{attacks}^{[15,16]}$.

1) idea: For every execution of the algorithm a fresh mask is randomly generated and applied to the input data and to the secret key ${ }^{[17]}$. All internal computations are masked from thereon and the final result is unmasked after the last round. In this case, the attacker cannot extract any correlation between the secret key in use and the actual power curves he is recording from each run of the algorithm.

2) evaluation: masking strategy has been widely used in resistance against side channel attacks. Unfortunately, there are some distinct disadvantages: firstly, attackers can filter random disturbing info, unless each bit is split into $\mathrm{k}$ shares using any scheme which has the required stochastic properties ${ }^{[18]}$; secondly, masking often need a very large memory space, and this will make key path larger than usual implementations, so that designers and users often have to balance among the designing cost, run speed and security; thirdly, even designers have considered the size of storage, such as the implementation in ${ }^{[19]}$ to change SBOX design from look up table to logic circuits, there still existed the shortage because that it is impossible to disturb all "zero-value" during multiplication in finite field ${ }^{[20]}$.

\subsection{Balancing}

Basing on complementary logics such WDDL-AND, Masked-AND[21,22] etc, balancing technologies have been proposed tried to keep the power of whole circuits at constant value to prevent DPA attacks.

Whenever an operation is performed in hardware, a complementary operation should be performed on a dummy element to assure that the total power consumption of the unit remains balanced according to some higher value.

Until now, there are some technologies based on balancing idea, such as Dual-rail Logic ${ }^{[23]}$ and complementary logics ${ }^{[24]}$.

Quantitative evaluation of leakage model is the precondition for balancing strategies. From the results of these leakage models ${ }^{[25]}$, even some countermeasures such as WDDL complementary logics which have shown almost constant power value in leakage model experiment results, will show small peaks when improving precision of evalutation. 
Besides small peaks, existing balancing countermeasures have contrained by EDA development kit, which means balancing countermeasures have to use standard circuit designing base and developing language, however, these conditions have not been totally satisfied. Moreover, balancing countermeasures will definitely increase hardware resources at least one time.

\subsection{Random Timing}

1) purposes:

Random timing belongs to randomizing countermeasure which is one of the most popular countermeasures against SCA attacks. Designers randomly introduce temporal jitter into the sequence of operations ${ }^{[8]}$ or change clock frequency ${ }^{[9]}$. They think that such countermeasures are effective because the intermediate result $f_{k c}(X)$ is no longer computed at a fixed instance. It rather occurs at a set of different time instants $T$ with probability distribution $P\left(f_{k c}(X)\right.$ and $P$ are all complied with model in kocher[2]). In fact, both random timing and changing clock frequency technologies hope to raise the cost of an attack beyond the gain due to a success. And a metric for measuring the difficulty of an attack is the number of samples required.

\section{2) Evaluation:}

We evaluate random timing technologies as following:

Advantages: this countermeasure has a simple designing idea, very convenient to implement, and limited hardware resource increasement. It also induced dynamic FPGA chip reconfiguration technology ${ }^{[26]}$ to chip designing which can make circuits reconfigurable when they are working ${ }^{[8]}$.

Disadvantages: however, there may be some insufficiency in random timing resistances.

First: this countermeasure only relies on working time of circuits. It is worth mentioning that timing is one character of data collection in DPAs, which means that an adversary can verify each round or each operation in circuits of cryptographic algorithms through collecting enough power samples. For example, hardware implementation of block cipher (such as $\mathrm{DES}^{[2]}$ ), attacker can not only fixed each round but each operation in each round through power samples. So, attacker can confirm the location of each random timing jitter which is the base of random timing countermeasures.

Second: even if attacker can not find the real locations of random timing jitters (as researchers stated 
in ${ }^{[8]}$ ), we simply analyzed the influence of jitter locations for each round of block cipher. Table 1 shows the success ratio against DPA attacks of each round.

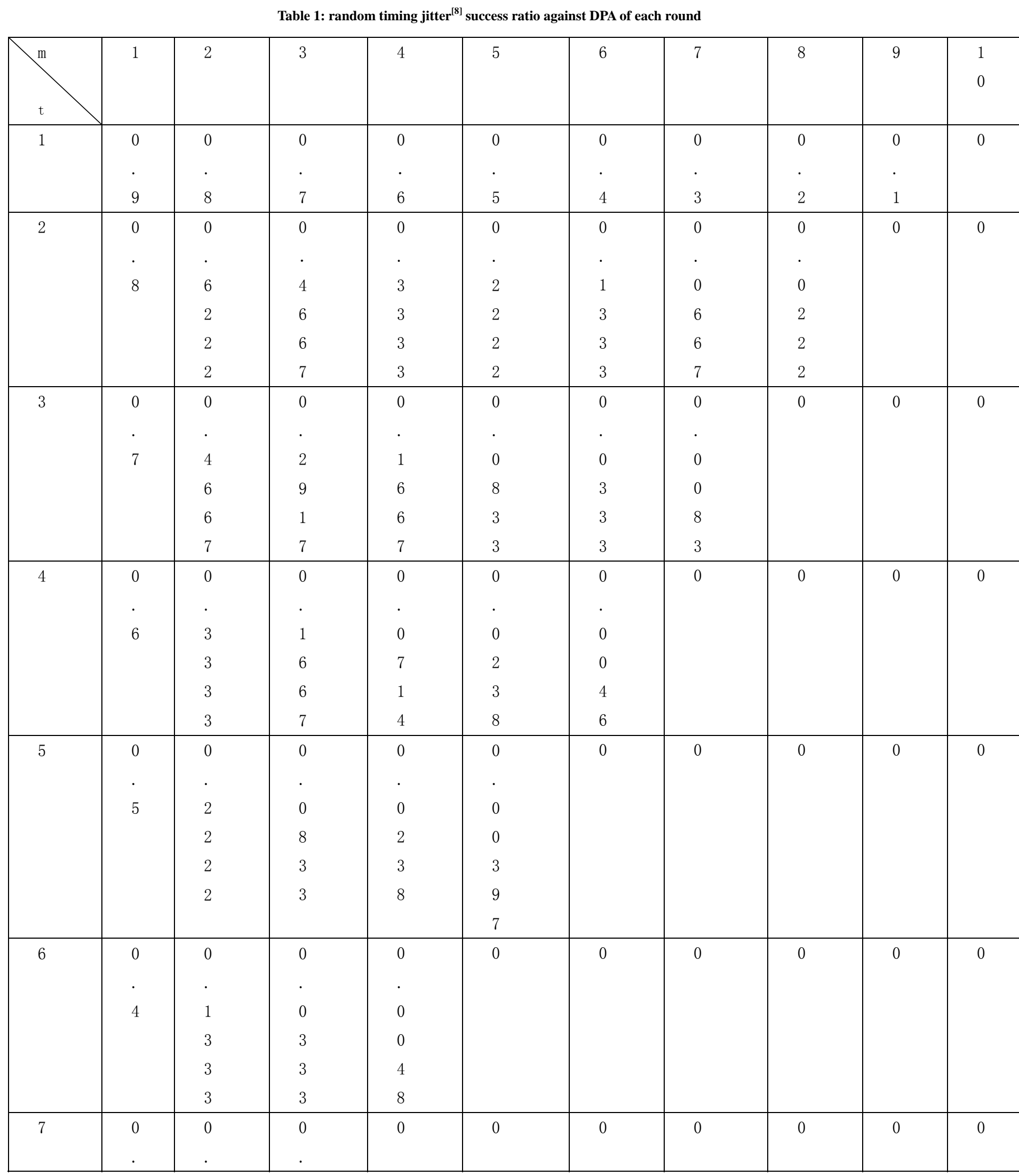




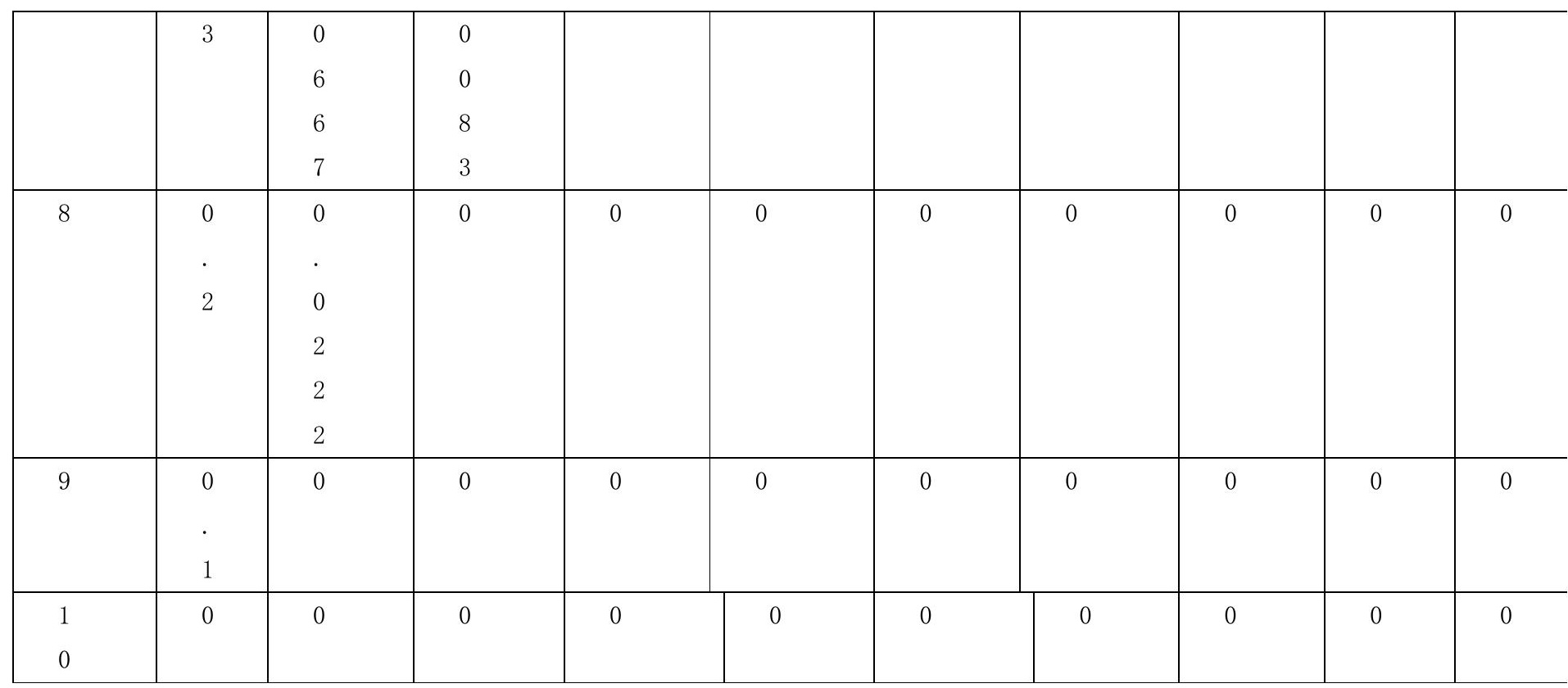

\section{Illumination:}

- $\mathrm{m}$ is the number of random timing jitters;

- $\mathrm{t}$ means the number of concerning points. In AES algorithm, “ $\mathrm{t}=10$ ” means each round corresponds a sample point;

- $\quad p_{i j}$ means success probability against DPA attack at the $\mathrm{j}^{\text {th }}$ sample point after injecting the $\mathrm{i}^{\text {th }}$ timing jitter.

\section{For example:}

Supposing $\mathrm{m}=\mathrm{i}$, and the condition of DPA attack in the $\mathrm{j}^{\mathrm{th}}$ sample point is i timing jitters are all injected after the $\mathrm{j}^{\text {th }}$ point, so that timing jitter can not influence the $\mathrm{j}^{\text {th }}$ point operation time. We suppose only if jitter injected before one round, then the power sample of this round will be disturbed and DPA attack will succeed. And we don't consider other situations such as inject the same number of jitters in each round. Then the probability of DPA attacks is shown as followed:

$$
P_{i j}=C_{n-j}^{i} / C_{n}^{i} \quad(i=1 \cdots m, j=1 \cdots t)
$$

The results in table 1 are the most common situation, and the success ratio of DPA can be increased when attackers make deeper analyzing.

Third: based on our experiments of AES algorithm, injecting timing jitter( or register in hardware implementation) in some round and operations may bring the variety of pre-round to post-round's registers which are injected as timing jitters, and this may magnify the value of power in these registers, for this can increase the reverse ratio of registers. If attacker can concern register power 
(some EDA tools provide this function ${ }^{[27,28]}$ ), timing jitter may provide more opportunities to DPA attacks.

So, randomly injecting timing jitter can not effectively resist against DPA attacks. Fortunately, this measure $^{[8]}$ is still an efficient countermeasure against CPA(Correlation Power Analysis) which is much closer with timing than DPA.

Random timing technology give us inspiration that dynamic circuit may increase complexity of DPA analyzing or other SCA attacks. In session 4, we will give a new dynamic cryptographic algorithm countermeasure against DPA attacks.

\section{Evolutionary Cipher against DPA}

After analyzing the existing countermeasures against DPAs, we find there are a few resistances using dynamic circuits to prevent DPA attacks. For example: masking strategy randomly selects masking code, which is random sequence generated with RNG(random number generator) or TRNG(true random number generator); random timing countermeasure also uses RNG(or TRNG) to randomly change clock frequency or locations of inject timing jitters. Besides these dynamic designing measures, now some chip vendors have provided dynamic reconfiguration technologies to configure circuits online ${ }^{[26]}$.

Existing dynamic designing measures have tried to making DPA attacks more difficulty and ideally infeasible. However, these dynamic disturbing countermeasures only have increased the complexity of DPA analyzing. We try to propose a new dynamic design measure which has the ability to destroy the base of DPA analyzing. It is worth to notice that our proposal could also effectively resist several mathematic analysises and has better scalability than usual countermeasures.

\subsection{Designing Principles of Evolutionary Cipher}

This paper tries to propose an effective countermeasure against DPAs, in fact, the purpose of evolutionary cipher is to resist different kinds of attacks of cryptographic attacks which include some SCA attacks and mathematic analyzing(off line attacks). We focus on block cipher designing and 
choose AES algorithm as original algorithm to evolve.

There are some characters of evolutionary cipher as following.

First: dynamic structure of cryptographic algorithm and implementation;

Second: Higher levels of security. The purpose of evolutionary cipher is to design safe cryptographic algorithm and to resist against different attacks;

Third: more efficient to design a new safe cryptographic algorithm than random or robust selecting;

Forth: complying with cryptographic algorithm designing principles;

Fifth: more scalablity than usual cryptographic algorithm, for evolutionary cipher can change evolutionary strategies on-line or off-line and even can be controlled by special designer or user.

In order to keep these characters, evolutionary cipher has the common structure as figure 1.

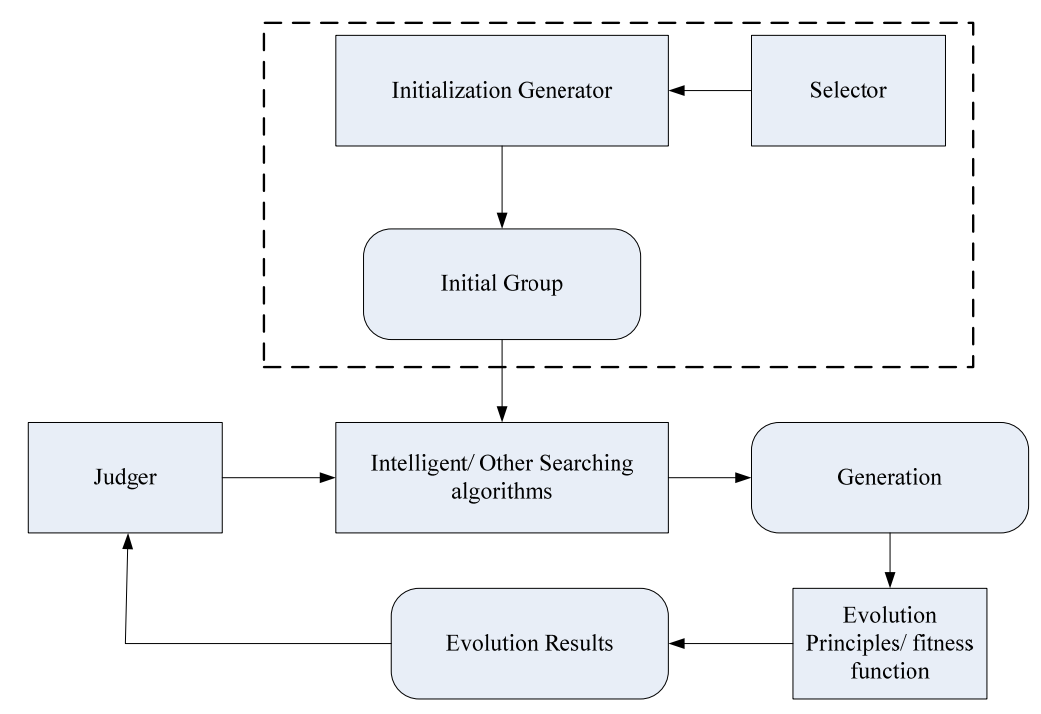

Figure 1: common structure of evolutionary cipher

\section{Illumination:}

- Selector and judger control the changing direction of evolutionary cipher. Both of them are controlled by the safe TRNG.

- Fitness functions are composed with different evaluations of security, and test each generation of evolutionary cipher.

- Evolutionary cipher combines intelligent searching algorithm and block cipher principles to efficiently and effectively design safe cryptographic algorithm. And these can be implemented in FPGA chips with dynamic reconfiguration technology ${ }^{[26]}$.

The detail and effectiveness against mathematic analyzes of evolutionary cipher have been published in several paper ${ }^{[10,11]}$ and Patent ${ }^{[29]}$. We conclude the flow of evolutionary cipher as following. 
Step1: initialization concludes initial group building, selection principles, judgment strategies and parameter configuration.

Step2: intelligent searching next generation is constrained by fitness function.

Step3: evaluating the new generation and this is the next key point after intelligent searching. Because there are several security evaluations, we should balance among these principles, which equal to solve multi-purpose problem.

Step4: if the output is not satisfied, process will go back to step1 or step2 based on designing strategies. This condition of output may be constrained by security evaluations or execution time.

Step5: if output condition is satisfied, new generation replaces present one, and dynamic reconfiguration technology can be used to implement evolutionary cipher in chips.

We choose SBOX of AES algorithm to introduce how evolutionary cipher resists against DPA attacks in 4.2 .

\subsection{Evolutionary Cipher against DPAs}

\subsubsection{Basic Idea}

We introduce evolutionary cipher which changes algorithm and logic implementation to resist DPA from collection and analyzing phases. In collection, dynamic implementation induces disturbing value of power; in analysis, evolutionary cipher destroy the differential power computing model proposed by kocher ${ }^{[2]}$.

In order to explain evolutionary cipher's resistance against DPAs, let's recall kocher's differential power computing model in session 2(researchers in Motorola proposed an other model ${ }^{[2]}$ which has the same principle as kocher).

Supposing: A is a cryptographic algorithm, D is the function based on kocher's model to compute the $b^{\text {th }}$ bit in input of last round in A.

When A has been changed, D will be changed as well. The key problem is whether attacker could find the details of changed D? And this problem equals whether attackers could find the details of changed A?

We classified these problems into two situations. First, it is still public structure of changed A, which means attackers can get every detail of changing results; second, attackers could find that A 
has been changed (for example, same plaintexts get different ciphertexts), but the specific structure of changed A is secret.

\subsubsection{Secrecy of Evolutionary Cipher}

The first situation in last part may be regarded as more comply with public designing principle of cryptographic algorithm. However, after deeply analyzing, we find it is reasonable for evolutionary cipher and some other dynamic algorithm to keep secret of their changing or changed results.

There are two types of operations in evolutionary cipher, and we call them static and dynamic structure parts respectively. In figure 1, static parts include intelligent generator, initial group generator and fitness functions; dynamic parts include selector and judger, which are controlled by TRNG.

Because we use TRNG to control dynamic structure of evolutionary cipher, the next generation can not be participated or controlled by designers, users or attackers, and this point has been coincided with other dynamic design such as random timing ${ }^{[8]}$. Moreover, we make every detail of evolutionary cipher public to ensure no backdoor in algorithm or implementation. In our opinion, public designing demanding of cryptographic algorithm is trying to stop secret in designing algorithm, but the secrecy of evolutionary cipher is to keep secret of designing results. This secrecy can also ensure real variety and non-intervention.

So, attackers or even designers of evolutionary cipher can not get knowledge of any evolved generation, and then can not build correct function of D. Unknown D, it is impossible for attackers to make an effective DPA attacks which are based on differential computation model proposed by kocher.

The reason why evolutionary cipher can resist DPA attacks is attackers can not build correct function D. Conversely, if attackers could construct correct D function after evolution, evolutionary cipher can not resist against DPAs. We will analyze the probability of losing resistance at 4.3.

\subsection{Security of Evolutionary Cipher}

In order to explain security of evolutionary cipher, we illustrate it from several aspects. 


\subsubsection{When attackers only know the details of evolutionary cipher, whether they can infer certain generation of algorithm?}

Because evolutionary cipher is controlled by TRNG, supposing that SBOX is changed by evolutionary cipher ${ }^{[29]}$, each changed value will be selected with same probability. For example, SBOX in AES has the same ratio of $1 / 256$ ! for each candidate (considering inverse operation). It is infeasible for attackers to successfully analyze parameter of SBOX within reasonable period of time. So we consider that this situation will be safe to resist DPA attacks.

\subsubsection{When attackers could get certain generation of evolutionary cipher, whether they can infer pre or post generation of algorithms?}

In this situation, attackers can get certain point of evolutionary cipher algorithm, for example, original AES algorithm. We borrow the concepts of "forward security and behind security" in digital signature to illuminate the safety problem existed in this situation.

Basing on the common structure of evolutionary cipher in figure 1, variety of evolutionary cipher originates from TRNG, and this is coincided with the idea proposed by Shannon ${ }^{[30]}$ that cryptographic algorithm security based on key. The difference is both key and algorithm are dynamic in evolutionary cipher.

We use following model to describe an evolutionary cipher system.

$$
S F_{g i}=\left(F G_{g i}, \text { trng }, e f\right)
$$

$S F_{g i}$ - means the $\mathrm{gi}^{\text {th }}$ generation of evolutionary cipher;

$F G_{g i}$ - means the initial group for the $\mathrm{gi}^{\mathrm{th}}$ generation;

trng - means random sequence generated by TRNG;

ef - concludes all evolutionary design principles

Among these components in function (3), only $F G_{g i}$ is related with time/generation, others are all controlled by TRNG. Therefore, security of $F G_{g i}$ is the key problem which needs to analyze.

We analyze security of evolutionary cipher from 2 aspects: first, the influence of random 
sequence length on the security of evolutionary cipher; second, forward and behind security of evolutionary cipher.

\subsubsection{Length of Random Sequence}

TRNG plays an important role in evolutionary cipher, and there are 2 properties of security TRNG, first, non-participated with true seed; second, impossible statistical analyzing with safe random network.

However, limited length of random sequence will give chance to attackers using robust search to find details of generation. In fact, there are two questions need to solve. One, how long is safety for random sequence of evolutionary cipher? The other, whether does the existing evolutionary cipher have a safe length of random sequence?

1) safe length of random sequence

Basing on information theory proposed by Shannon ${ }^{[30]}$, longer key brings more security of cryptographic algorithm. In theory, one-time system is a real safe situation. Therefore, if there is no limitation for random sequence length, it is the real safe situation as well. Unfortunately, constrained by present operation and storage resource limitation, existing evolutionary cipher can not use unlimitedly long random sequence.

Standing on attacker's point of view, it may be easier to find safe length of random sequence. Supposing that key is constant during DPA attacks, if key changed, attackers will make a new attack from scratch. So, we can make such hypothesis that it is safe if random sequence does not repeated unless key changed. For the longest cycle of key is in line with the length of key, the length of random sequence must not be smaller than that of key. Such as 128bits key AES algorithm, the length of random sequence in evolutionary cipher will not be less than 128bits.

In conclusion, for security of evolutionary cipher, the length of random sequence will not be smaller than that of key in original cryptographic algorithm.

2) length of random sequence in existing evolutionary cipher

After knowing the safe length of random sequence, we analyze whether did the design of evolutionary SBOX reach safe level at the point of random sequence? Such as SBOX in AES algorithm, we have designed evolutionary SBOX in a number of $256 !\left(256 !>2^{128}\right)$ which means SBOX evolutionary design ${ }^{[29]}$ is safe with long enough random sequence. In order to reach safe level, 
it should choose effective evolutionary objects which have rich enough candidates.

\subsubsection{Forward and Behind Security of Different $F G_{g i}$}

$F G_{g i}$ is generated with initial group generator. Certain $F G_{g i}$ may be a totally new one, or partly selects from $F G_{g i-1}$. So, there maybe existing some relationship between neighboring $F G_{g i-1}$ and $F G_{g i}$. This kind of relationship may create association among generations of evolutionary cipher, therefore, forward and behind security of $F G_{g i}$ is the key security problem.

We use the theory of "cipher text independence on plaintext" to quantitatively analyze the relationship between neighboring generations. $S F_{g i}$ and $S F_{g i+1}$ are neighboring evolutionary cipher algorithms and are regarded as plaintext and cipher text respectively. When cipher text is independent on plaintext, it is true that plaintext is independent on cipher text.

Firstly, we introduce the test of independence between plaintext and cipher text. Supposing the size of one block in cryptographic algorithm is $\mathrm{n}$ bits, we randomly select $\mathrm{F}$ blocks of plain text $P, P_{1}, \cdots, P_{\mathrm{F}-1}$ and one key. Under the mode of ECB, we encrypt plaintexts with the key and outputs F blocks of cipher text $C_{0}, C_{1}, \cdots, C_{F-1}$, and then compute distances between plaintext and cipher text: $\quad D_{i}=W\left(P_{i} \oplus C_{i}\right), 0 \leq i \leq F-1$ where $\mathrm{W}$ means hamming weight between plaintext and cipher text. The number of $D_{i}(0 \leq i \leq F-1)$ equals $w(0 \leq w \leq n)$ will be called $H_{w}$.

For a safe block cipher, $H_{w}$ should be satisfied with the distribution of $B(n, 1 / 2)$. Using pearson $\chi^{2}$ to verify whether $H_{w}$ is satisfied with $B(n, 1 / 2)$. The expectation of $H_{w}$ is $E_{w}=C_{n}^{w} \times F / 2^{n}$, then $\chi^{2}=\sum_{i=0}^{n} \frac{\left(H_{i}-E_{i}\right)^{2}}{E_{i}}$. To comparing the threshold when pearson fitting is $\chi^{2}$, significance level is $\alpha$ and freedom is $n$, if $\chi^{2}<\chi_{\alpha}^{2}(n)$. It is proofed that ${ }^{H} w$ is satisfied with $B(n, 1 / 2)$, and correspondingly, plaintext and cipher text are independence, otherwise, they are dependable.

During experiments, we regard known cryptographic algorithm A(original SBOX in AES algorithm) as plaintext, and any generation behind A called $A^{\prime}$ as cipher text. The results of 
experiments are shown as table 2 .

SBOX in $A^{\prime}$ (decimal representation)

167, 204, 144, 11, 225, 182, 57, 159, 209, 231, 32, 109, 30, 90, 37,215 ,

$33,236,227,40,214,97,98,43,220,222,124,71,198,81$, 174,21 ,

$210,48,67,26,181,17,106,50,149,171,130,237,228,122$,

148,128 ,

$108,28,12,154,85,189,186,176,129,249,136,247,47,178$,

217,234 ,

$161,142,162,42,197,80,194,86,211,218,180,179,133,22$,

113,35 ,

$239,243,121,201,56,41,103,125,75,199,244,29,96,52$, 34,100 ,

147, 58, 104, 200, 251, 143, 82, 250, 169, 123, 44, 70, 1, 84, 160,172 ,

5, 138, 7, 207, 9, 131, 27, 0, 246, 140, 168, 69, 116, 141, 3,233 ,

76, 15, 152, 45, 38, 156, 137, 229, 202, 230, 252, 253, 19, 16, 78,184 ,

126, 254, 240, 91, 101, 146, 115, 193, 39, 221, 92, 102, 135, 72, 8,139 ,

120, 99, 163, 166, 63, 87, 127, 132, 183, 110, 238, 111, 188, 62, 73,173,

$46,107,51,190,157,49,224,155, \quad 4,158,203,118,205,255$,

36,20 ,

$223,114,83,61,60,74,191,64,177,196,66,219,145,65$

165,248 ,

119, 212, 185, 134, 213, 53, 55, 25, 170, 105, 216, 24, 94, 175,

$31, \quad 13$,

$151,153,95,89,23,226,187,117,112,206,195,242,192,68$,

150,245 ,

235, 164, 59, 208, 2, 93, 79, 88, 18, 54, 10, 14, 232, 6,

$241, \quad 77$

Table 2: experiments of $H_{w}$ between different evolutionary SBOXes

\begin{tabular}{|l|l|l|l|l|l|l|l|l}
\hline $\mathrm{w}$ & 5 & 5 & 5 & 5 & 5 & 5 & 6 & 6 \\
\hline$H_{w}$ & 4 & 5 & 6 & 7 & 8 & 9 & 0 & 1 \\
\hline
\end{tabular}




\begin{tabular}{|l|l|l|l|l|l|l|l|l}
\hline $\mathrm{w}$ & 6 & 6 & 6 & 6 & 6 & 6 & 6 & 6 \\
\hline$H_{w}$ & 2 & 3 & 4 & 5 & 6 & 7 & 8 & 9 \\
\hline
\end{tabular}

As for other values of $\mathrm{w}, H_{w}$ are all zero. Because $\chi^{2}$ is 20.1 which is smaller than $\chi^{2}$ at $\alpha$ $=0.05$ and $\alpha=0.1, S F_{g i}$ (plaintext) and $S F_{g i+1}$ (cipher text) are proofed to be independent.

Table 3 shows values of $\chi^{2}$ among different 10 generations of evolutionary SBOXes.

Table 3: experiments of $\chi^{2}$ between different evolutionary SBOXes

\begin{tabular}{|c|c|}
\hline SBOXes & $\chi_{\text {value }}^{2}$ \\
\hline $\mathrm{s} 0-\mathrm{s} 1$ & 20.1 \\
\hline $\mathrm{s} 0$ - s2 & 14.3 \\
\hline s0-s3 & 29.1 \\
\hline $\mathrm{s} 0-\mathrm{s} 4$ & 22.8 \\
\hline s0—s5 & 50.5 \\
\hline s0—s6 & 46.2 \\
\hline s0—s7 & 20.3 \\
\hline s0—s8 & 69.8 \\
\hline s0—s9 & 19.4 \\
\hline s1—s2 & 27.8 \\
\hline s2-s3 & 21.6 \\
\hline s3-s4 & 13.7 \\
\hline s4-s5 & 20.5 \\
\hline s5-s6 & 15.6 \\
\hline
\end{tabular}

S0 is the original SBOX in AES algorithm, and S1...S9 are generated with evolutionary cipher $^{[29]}$. Appendix1 shows the values of these evolutionary SBOXes, and appendix2 introduces the theory and flow of evolutionary cipher ${ }^{[29]} . \quad \chi_{0.05}^{2}(128)=154.3015159 \quad \chi_{0.1}^{2}(128)=147.8048$

We have made extensive experiments and tested any two boxes among 100 evolutionary 
SBOXes, and only found 4 couples not to be satisfied with indenpent theory. So we have set independent theory in evolutionary cipher to guarantee security of EVOC.

\section{Experiments of Evolutionary Cipher Effectively against DPAs}

Different with last experiments, the experiments in this section are trying to test the effectiveness of EVOC resistance against DPA attacks.

In order to decrease noise disturbing from test equipments and circuits, we adapted cyclone2 series FPGAs of Altera as test chips, DE2-70FPGA development kits and primepower of synopsys as test environments.

We designed three different levels of experiments to test the resistance of evolutionary cipher against DPAs.

The follow of experiments is shown as figure 2.
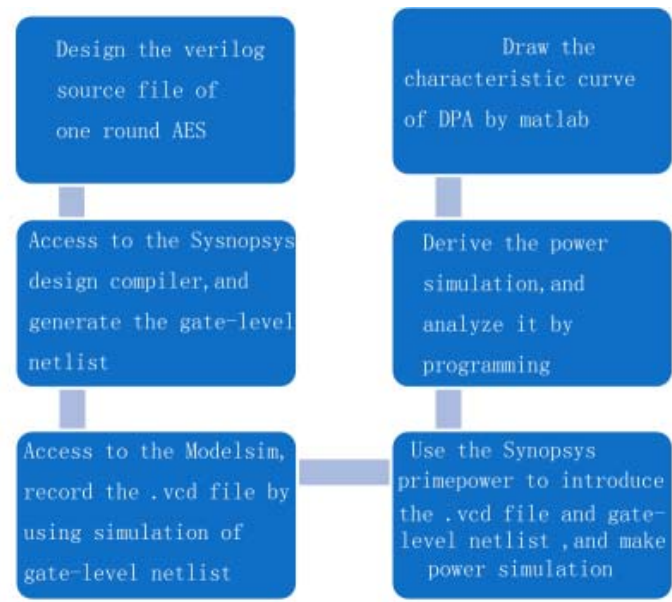

Figure2: the follow of experiments

\subsection{TEST1}

\subsubsection{Test1 Measure}

1) test purposes: When attackers have known every detail of D function whenever it changed, it tests whether evolutionary cipher can resist against DPA attacks effectively.

2) parameters and conditions:

- Known: plaintext P, key Ks, and Ks keep unchanged during DPA attacks; 
- Attackers can put correct Ks into differential power computation model[kocher]. And if it shown peaks with correct Ks, resistance is not success;

- Making extensive experiments to calculate success ratio.

3) Test1 Steps:

Step1: Randomly selects plaintext P and key Ks, Ks keeps unchanged during DPA attacks, while P changs randomly in every test;

Step2: Power sample point is set at the end of first round of AES algorithm;

Step3: Using EDA simulation and power analyzer to collect certain information such as cipher text $C_{i}$ and power $T_{i}[j]$ on line;

Step4: To put correct Ks into D function and compute value of b. The reason why we can use correct Ks is that test1 supposes attackers can get every detail of cryptographic algorithm which means that attackers can get details of D function. If correct Ks is collected by attacker, the value of b is correct. The real purpose of test 1 is to find whether does power peaks in simulation tools. In order to decrease the complexity of test, we directly bring correct Ks into D to compute correct b.

Step5: To compute differential power based on the model proposed by kocher, and judge whether the condition of DPA is satisfied.

Step6: Making extensive experiments to calculate success ratio.

\subsubsection{Analysis of Test1}

The results are listed in figure 3 and 4.

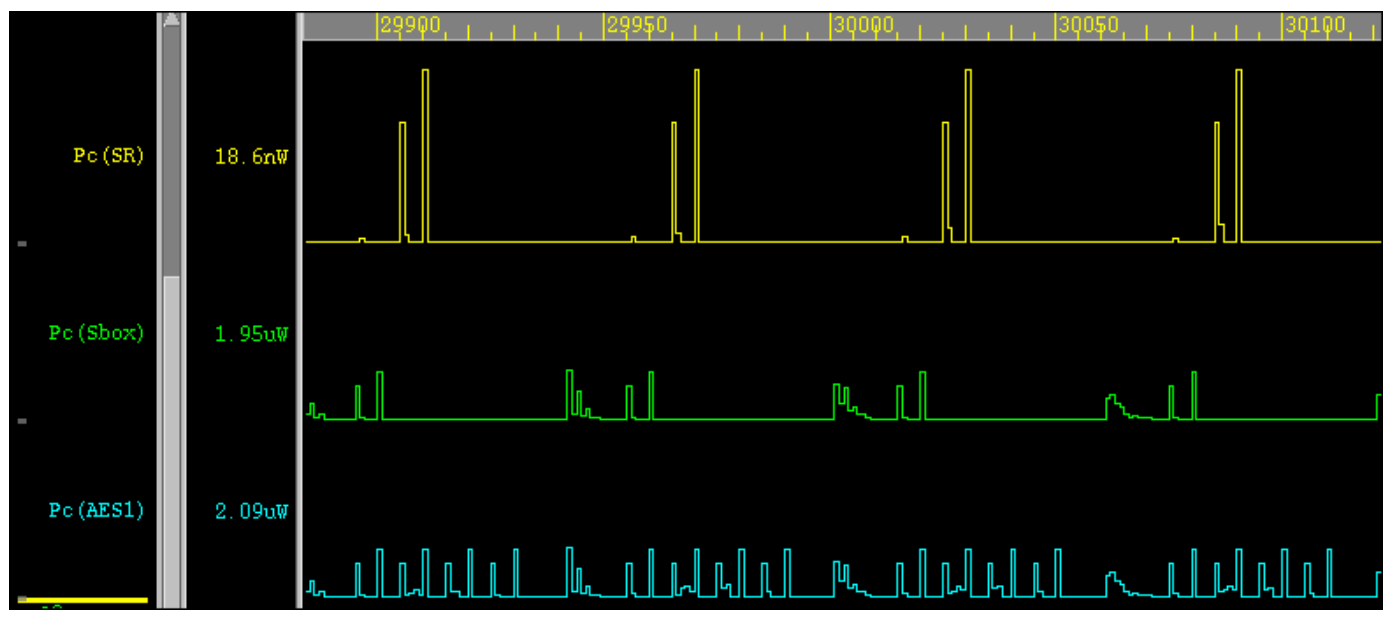

Figure 3: experiment results of test 1 in primepower 


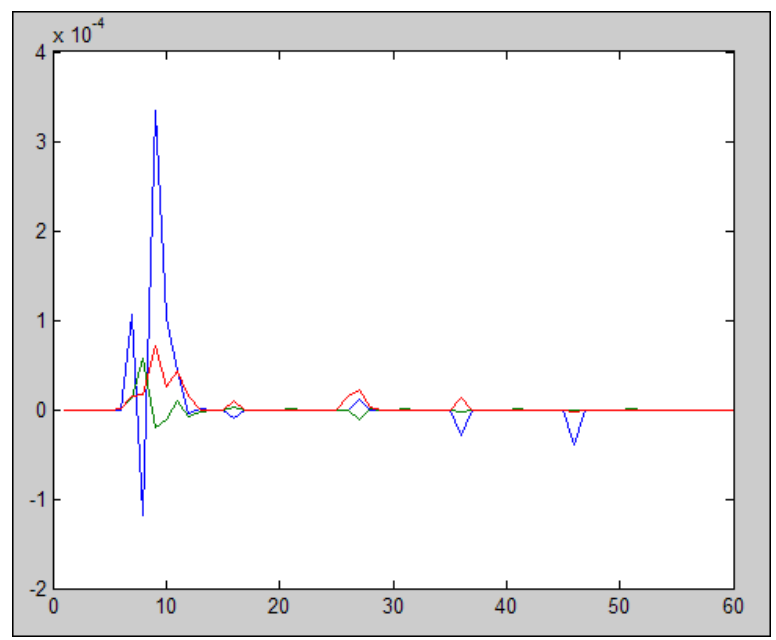

Figure 4: power cycles of test1 in matlab

Illustration:

\begin{tabular}{|c|c|c|c|c|c|c|}
\hline Test & $\begin{array}{l}\text { Number } \\
\text { of } \\
\text { samples }\end{array}$ & $\begin{array}{l}\text { Time } \\
\text { of } \\
\text { test }\end{array}$ & $\begin{array}{l}\text { Blue } \\
\text { cycle } \\
\text { (attackers } \\
\text { know) }\end{array}$ & $\begin{array}{l}\text { Gree } \\
\text { n } \\
\text { cycle }\end{array}$ & $\begin{array}{l}\text { Red } \\
\text { cycle }\end{array}$ & conclusion \\
\hline Test1 & 10,000 & $60 \mathrm{~ns}$ & $\begin{array}{l}\text { Correct } \\
\text { traces }\end{array}$ & $\begin{array}{l}\text { Ran } \\
\text { dom } \\
\text { trac } \\
\text { es }\end{array}$ & $\begin{array}{l}\text { Origin } \\
\text { altrace } \\
\text { s }\end{array}$ & DPA Success \\
\hline
\end{tabular}

The results of test 1 have shown that if attackers can get knowledge of every detail of $D$ function or cryptographic algorithm, attackers can find blue cycle which represents correct trace, so DPA can be success. Moreover, effectiveness of DPA dependents on the number of samples, more samples more effective.

\subsection{TEST2}

\subsubsection{Test2 Measure}

1) test purposes: Attackers can find D function has been changed, but don't know the details of variety. Test2 evaluate the success ratio of evolutionary cipher resistance.

2) parameters and conditions: Attackers regard D as randomly changed and conditions are same as test1. 


\section{3) Test2 Steps:}

Step1: Randomly selects plaintext P and key Ks, Ks keeps unchanged during DPA attacks, while P changes randomly in every test;

Step2: Power sample point is set at the end of first round of block cipher such as AES algorithm;

Step3: Using EDA simulation and power analyzer to collect only power $T_{i}[j]$ on line;

Step4: For attackers do not know the details of changed D, they regard D as random function, and then randomly select the value of $b$ as each power cycle;

Step5: To compute differential power based on the model proposed by kocher, and judge whether the condition of DPA is satisfied.

Step6: Making extensive experiments to calculate success ratio.

\subsubsection{Analysis of Test2}

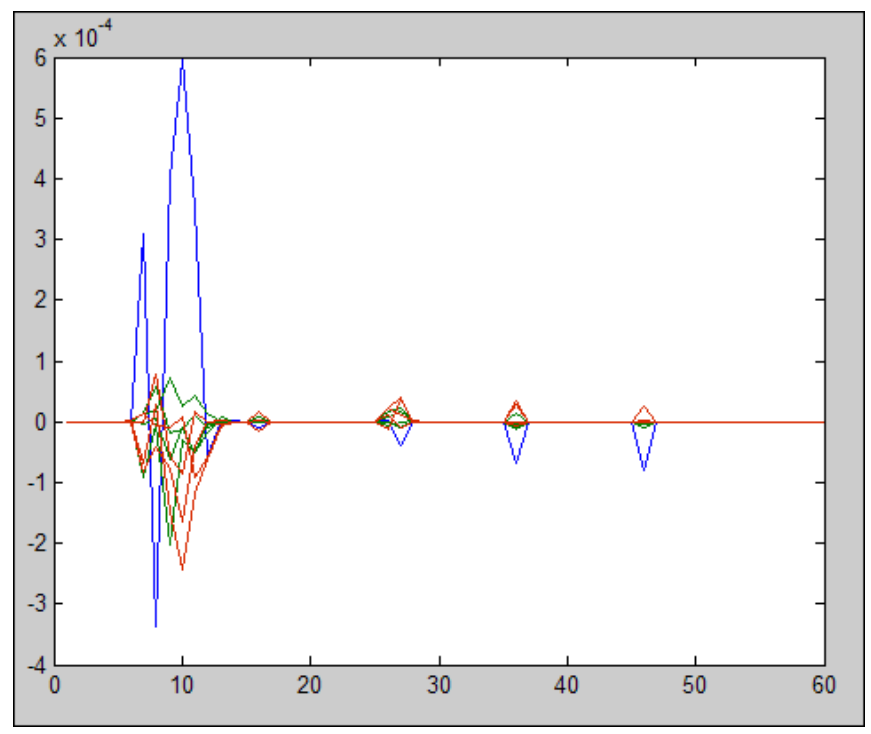

Figure 5: power cycles of test2 in matlab

\section{Illustration:}

\begin{tabular}{|l|l|l|l|l|l|l}
\hline Test & Number & Time & Blue & Green & Red & conclusion \\
& of & of & cycle & cycle(att & cycle & \\
& samples & test & & kckers & & \\
& & & know) & & \\
\hline Test2 & 10,000 & $60 \mathrm{~ns}$ & Corr & Rando & Origin & DPA Failure \\
\hline
\end{tabular}




\begin{tabular}{|l|l|l|l|l|l|l|}
\hline & & ect & mtraces & altrace & \\
trac & es & & \\
\hline
\end{tabular}

In test2, attackers can not get hold of correct traces, but get random traces (green), so the DPA attacks are fail.

\subsection{TEST3}

\subsubsection{Test3 Measure}

1) test purposes: Attackers don't find the variety of $D$ function, and use original $D$ function to compute differential power. Test3 tries to calculate the success ratio of evolutionary cipher resistance against DPAs.

2) parameters and conditions: Parameters and conditions are same as test1.

3) Test3 Steps:

Step1: Randomly selects plaintext P and key Ks, Ks keeps unchanged during DPA attacks, while P changes randomly in every test;

Step2: Power sample point is set at the end of first round of block cipher such as AES algorithm;

Step3: Using EDA simulation and power analyzer to collect certain information such as cipher text $C_{i}$ and power $T_{i}[j]$ on line;

Step4: For attackers have not found the variety of $\mathrm{D}$ function, test3 puts observed cipher text $C_{i}$ and correct key Ks into D function to compute b corresponded with each power cycle.

Step5: To compute differential power based on the model proposed by kocher, and judge whether the condition of DPA is satisfied.

Step6: Making extensive experiments to calculate success ratio. 


\subsubsection{Analysis of Test3}

The results of test 3 are also shown in figure 5, and attackers can get red power cycle which represents original traces and has no relationship with correct traces, so the DPA attacks are failure too.

\section{4 test 4}

From previous tests, we can find that SBOX plays an important role in DPA attacks. If attackers can not get knowledge of SBOX, DPA attackers can not be success. However, for DPAs have timing property, if attackers can observe linear operations in one round of AES algorithm, they could not regard of SBOX. Test 4 is designed to test whether DPA attacks on linear operations can success. Results of test 4 are showed in figure 6 and 7.

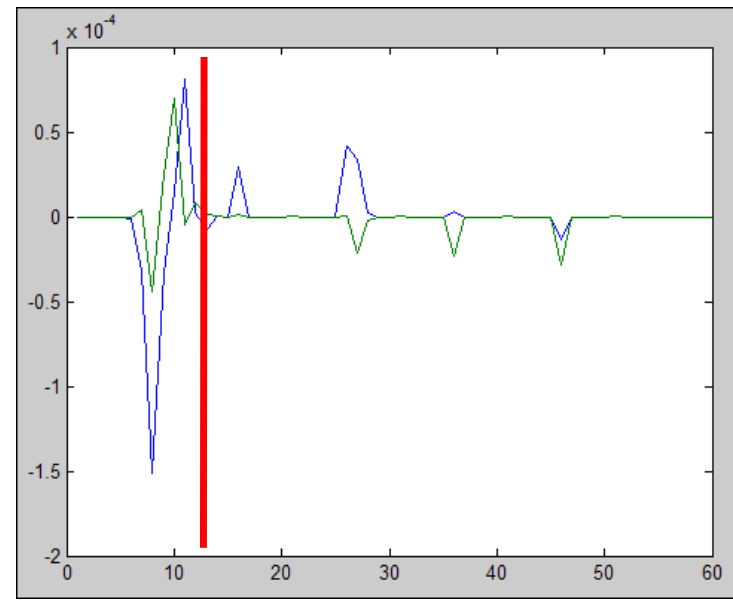

Figure 6: one round with SBOX

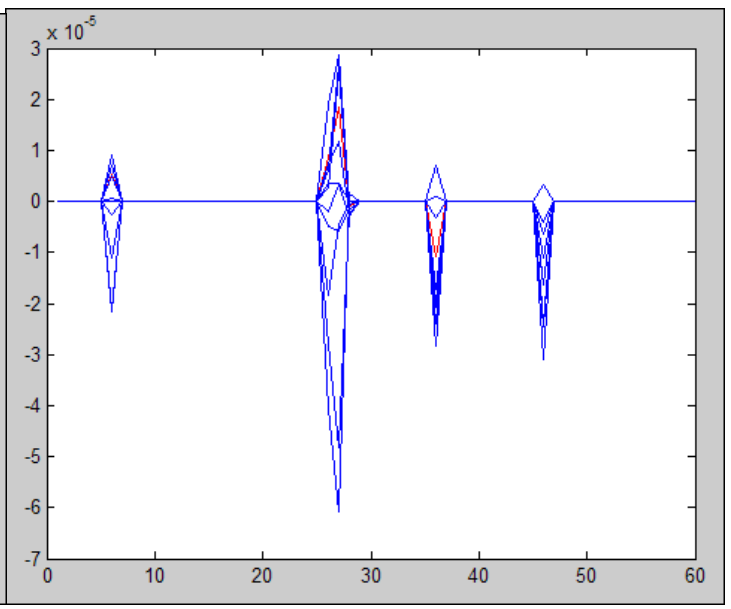

Figure 7:one round without SBOX

Figure 6 shows the results of one round implementation with SBOX, and the cycles behind red line represents the power consumed by linear operations. Blue and green cycles are correct and random traces respectively.

The maxmum value of blue cycles after red line are smaller than the right peak about 10 times, so this case DPA attack is ineffective.

Figure 7 shows the results of one round without SBOX, and red and blue cycles are correct and random traces respectively. For correct cycles are all covered by random ones, DPA attack is failure, too.

So, DPA attacks can not be success on linear operations of AES algorithms. 


\subsection{Analysis of Tests}

After security analyzing in session 4 and experiments in session 5, we can verify evolutionary cipher can effectively resist against DPA attacks from dynamic algorithm aspect. Comparing with some existing resistances, the purpose of evolutionary cipher is mainly focused on destroying differential power computation model in analyzing of DPAs. Using evolutionary cipher designing principles, cryptographic algorithms can effectively resist against DPAs through extensive experiments and are proofed to be a safety implementation to resist several attacks. Table 7 shows the combined results of different effective experiments.

Table 7: combined results of effective experiments

\begin{tabular}{|c|c|c|c|c|c|c|}
\hline Test & $\begin{array}{l}\text { Number } \\
\text { of } \\
\text { samples }\end{array}$ & $\begin{array}{l}\text { Time } \\
\text { of } \\
\text { test }\end{array}$ & $\begin{array}{l}\text { Blue } \\
\text { cycle }\end{array}$ & $\begin{array}{l}\text { Green } \\
\text { cycle }\end{array}$ & $\begin{array}{l}\text { Red } \\
\text { cycle }\end{array}$ & conclusion \\
\hline Test1 & 10,000 & 60ns & $\begin{array}{l}\text { Atta } \\
\text { cker } \\
\text { kno } \\
\text { w }\end{array}$ & & & DPA Success \\
\hline Test2 & 10,000 & 60ns & & $\begin{array}{l}\text { Attacke } \\
\text { r know }\end{array}$ & & DPA Failure \\
\hline Test3 & 10,000 & 60ns & & & $\begin{array}{l}\text { Attack } \\
\text { er } \\
\text { know }\end{array}$ & DPA Failure \\
\hline
\end{tabular}

Bule, red, green cycles are represents correct, random, and original traces respectively.

The reason why evolutionary cipher can effectively resist against DPA attacks is its dynamic structure controlled by TRNG which can generate both safety and unpredictability of random sequence. Safety random sequence generated by TRNG meets the Gaussian distribution and makes different power almost same in division of one or zero. Moreover, unpredictability of TRNG prevents attackers to get the details of correct algorithm and then can not acquire correct division of one and zero. This difficulty was also introduced in the research of DFA [31].

However, it should be particularly pointed out that TRNG is the key point of evolutionary cipher 
but is not as long as TRNG controls variety of algorithm can be against DPAs or other SCAs. Moreover, through extensive experiments, we find that only using TRNG to control the variety of evolutionary cipher can not fit the demand of security of cryptographic algorithm. As we introduce in section 4 and 5, safe TRNG must generate long enough random sequence and evolutionary objects must have large enough size. Focused on the base of DPA analyzing, this paper utilizes dynamic structure of evolutionary cipher to resist against DPAs. From this point of view, we combined TRNG, intelligent searching algorithm and cryptographic designing principles to automatically design cryptographic algorithms and EVOC can not only resist DPA attacks but several mathematic analyzes.

\section{Conclusion}

This paper proposes a new countermeasure called evolutionary cipher against DPA attack which is one of the most threatening in SCA attacks. It is firstly to combine intelligent computation method and cryptographic designing principles in design dynamic structure algorithm in order to resist DPA attacks. The advantage of evolutionary cipher is it can also resist several mathematic analyzes besides DPAs. EVOC provides a new idea for design and implementation of cryptographic algorithm which can achieve both cryptographic and physical security.

EVOC is to destroy the differential power computation model proposed by kocher. Moreover, we have quantitatively analyzed effectiveness and security of evolutionary cipher against DPA attacks, and concluded designing principles which can also be learned by other dynamic structure cryptographic algorithm to resist DPAs.

We will continue to deeply study designing methods and theory of EVOC, and the results will be applied to resist more SCA attacks and mathematic analyzes. At the same time, we will make an intensive study of the dynamic aspects of the algorithm.

\section{Appendix:}

\section{Appendix1:}

Evolutionary SBOXs tested in part 5.

s0: 
99, 124, 119, 123, 242, 107, 111, 197, 48, 1, 103, 43, 254, 215, 171, 118, 202, 130, 201, 125, 250, 89, 71, 240, 173, 212, 162, 175, 156, 164, 114, 192, 183, 253, 147, 38, 54, 63, 247, 204, 52, 165, 229, 241, 113, 216, 49, 21,

$4,199,35,195,24,150, \quad 5,154,7,18,128,226,235,39,178,117$,

$9,131,44,26,27,110,90,160,82,59,214,179,41,227,47,132$,

$83,209, \quad 0,237,32,252,177,91,106,203,190,57,74,76,88,207$, 208, 239, 170, 251, 67, 77, 51, 133, 69, 249, 2, 127, 80, 60, 159, 168, $81,163,64,143,146,157,56,245,188,182,218,33,16,255,243,210$, 205, 12, 19, 236, 95, 151, 68, 23, 196, 167, 126, 61, 100, 93, 25, 115, $96,129,79,220,34,42,144,136,70,238,184,20,222,94,11,219$, $224,50,58,10,73, \quad 6,36,92,194,211,172,98,145,149,228,121$, 231, 200, 55, 109, 141, 213, 78, 169, 108, 86, 244, 234, 101, 122, 174, 8, $186,120,37,46,28,166,180,198,232,221,116,31,75,189,139,138$, 112, 62, 181, 102, 72, 3, 246, 14, 97, 53, 87, 185, 134, 193, 29, 158, $225,248,152,17,105,217,142,148,155,30,135,233,206,85,40,223$, $140,161,137,13,191,230,66,104,65,153,45,15,176,84,187,22$

s1:

167, 204, 144, 11, 225, 182, 57, 159, 209, 231, 32, 109, 30, 90, 37, 215, $33,236,227,40,214,97,98,43,220,222,124,71,198,81,174,21$, $210,48,67,26,181,17,106,50,149,171,130,237,228,122,148,128$, $108,28,12,154,85,189,186,176,129,249,136,247,47,178,217,234$, $161,142,162,42,197,80,194,86,211,218,180,179,133,22,113,35$, 239, 243, 121, 201, 56, 41, 103, 125, 75, 199, 244, 29, 96, 52, 34, 100, $147,58,104,200,251,143,82,250,169,123,44,70,1,84,160,172$, 5, 138, 7, 207, 9, 131, 27, 0, 246, 140, 168, 69, 116, 141, 3, 233, 76, 15, 152, 45, 38, 156, 137, 229, 202, 230, 252, 253, 19, 16, 78, 184, 126, 254, 240, 91, 101, 146, 115, 193, 39, 221, 92, 102, 135, 72, 8, 139, $120,99,163,166,63,87,127,132,183,110,238,111,188,62,73,173$, $46,107,51,190,157,49,224,155,4,158,203,118,205,255,36,20$, 223, 114, 83, 61, 60, 74, 191, 64, 177, 196, 66, 219, 145, 65, 165, 248, 119, 212, 185, 134, 213, 53, 55, 25, 170, 105, 216, 24, 94, 175, 31, 13, $151,153,95,89,23,226,187,117,112,206,195,242,192,68,150,245$, $235,164,59,208, \quad 2, \quad 93, \quad 79, \quad 88, \quad 18, \quad 54,10,14,232, \quad 6,241,77$

s2:

5, 76, 187, 139, 159, 240, 177, 4, 202, 7, 84, 206, 119, 233, 188, 6, 204, 15, 13, 130, 254, 11, 155, 8, 37, 55, 109, 0, 21, 19, 29, 26, $47,89,216,141,106,78,235,247,50,92,46,68,191, \quad 3,179,169$, 156, 209, 137, 140, 48, 58, 246, 117, 225, 165, 128, 232, 154, 234, 248, 2, $182,244,107,59,111,151,51,146,239,69,180,201,110,193,16,105$, $189,243,66,142,63,194,27,250,72,207,211,251,172,113,49,83$, 121, 116, 149, 122, 98, 186, 129, 168, 162, 227, 229, 71, 167, 22, 123, 45, $62,88,131,85,86,94,236,1,126,152,148,173,133,150,170,161$, $38,12,99,242,220,30,42,238,93,174,215,200,103,95,96,120$, 
134, 25, 226, 40, 73, 217, 41, 57, 9, 104, 32, 124, 138, 171, 237, 102, 127, 24, 175, 198, 255, 54, 20, 74, 241, 52, 199, 28, 90, 212, 80, 245, $100,135,125,53,147,184,70,97,114,213,221,101,192,118,223,35$, 203, 249, 56, 143, 231, 164, 158, 160, 253, 64, 224, 43, 252, 144, 183, 163, $18,218,145,81,17,67,10,196,79,197,166,87,185,176,205,195$, $77,65,36,219,14,190,214,210,153,75,31,178,44,61,91,181$, $39,60,33,23,157,82,108,132,136,115,208,34,228,222,230,112$

\section{s3:}

164, 113, 250, 180, 14, 111, 167, 132, 124, 228, 86, 252, 66, 104, 26, 196, 188, 249, 185, 137, 178, 121, 142, 25, 208, 170, 37, 4, 158, 94, 131, 99, $141,235, \quad 6,116,197,49,40,143,10,75,173,108,122,100,231,128$, $110,59,244,84,74,23,175, \quad 2,117,29,201,72,174, \quad 8,114,68$, $71,239,229, \quad 55,101,19,42,179,168,76, \quad 7, \quad 28,69, \quad 1, \quad 62,165$, $58,15,172,20,183,97,67,50,241,220,123,18,32,130,106,182$, 159, 34, 83, 255, 216, 218, 233, 160, 253, 53, 245, 12, 93, 254, 223, 205, $151,203,169,118,22,11,200,36,127,238,115, \quad 0,105,51,224,157$, $176,153,248,47,134,227,45,136,107,96,251,60,120,43,152,191$, $9, \quad 3,21,109,209, \quad 38,77,119,57,133,112,63,148,192,232,88$, 95, 35, 64, 225, 146, 138, 190, 177, 79, 202, 193, 163, 139, 155, 214, 207, $24,41,31,234,147,154,44,184,226,187,166,56,33,98,230,16$, $92,82,87,52,181,61,46,189,210,236,85,13,242,243,103,221$, 126, 70, 211, 246, 30, 140, 89, 161, 17, 129, 125, 54, 186, 135, 156, 65, $81,204,240,102,217,90,219,91,206,145,195,199,237,247,171,39$, $144,215,80,222,78,150, \quad 5,73,212,194,27,48,213,198,149,162$

\section{s4:}

170, 217, 207, 234, 56, 161, 166, 42, 237, 183, 69, 215, 21, 189, 104, 55, $202,195,222,30,242,249, \quad 2,100,103,146,148,16,66,101,54,145$, $14,139,24,205,51,196,160, \quad 6,40,49,142,173,245,141,187,58$, $165,236,247,77,53,92,134,8,201,116, \quad 3,61,130,32,213,13$,

$1,155,179,220,137,76,168,246,154,45,28,112,9, \quad 4,248,174$, $232,60,138,80,230,153,17,200,227,87,241,72,128,50,181,226$, $70,136,81,219,71,79,131,186,211,212,243,48,105,223,91,19$, 102, 11, 158, 197, 88, 44, 7, 144, 225, 159, 209, 0, 185, 204, 167, 78, 250, 94, 199, 188, 34, 171, 180, 26, 177, 157, 203, 240, 253, 172, 90, 198, 36, 12, 84, 169, 99, 152, 41, 193, 228, 46, 221, 252, 106, 39, 135, 125, $97,140,29,163,114,18,194,254,33,15,35,182,22,86,127,27$, 96, 164, 124, 143, 118, 82, 176, 218, 175, 214, 162, 224, 132, 149, 191, 64, 109, $85,65,208,238,244,184,206,111,151,73,52,239,235,129,83$, 229, 5, 107, 255, 120, 10, 121, 190, 68, 62, 233, 216, 210, 38, 74, 25, 89, 23, 231, 133, 67, 117, 75, 113, 31, 126, 43, 59, 147, 251, 150, 156, 122, 123, 93, 95, 37, 98, 20, 57, 119, 47, 108, 192, 115, 63, 110, 178 
55:

146, 67, 27, 143, 224, 190, 162, 168, 147, 230, 9, 123, 84, 206, 189, 220,

15, 43, 95, 120, 239, 195, 8, 141, 129, 114, 106, 64, 21, 137, 216, 126,

$56,22,96,19,204,55,186,24,160,196,2,142,243,14,214,232$,

$174,151,251,41,212,109, \quad 34,32, \quad 3,205,12,244,50,128,115,52$,

$4,86,246,87,30,45,154,255,82,180,112,221,36,16,199,130$,

135, 240, 18, 93, 191, 94, 68, 7, 171, 65, 227, 61, 58, 200, 238, 175,

$5,26,89,75, \quad 1,33,54,210,107,119,235,192,185,91,113,76$,

$133,44,66,51,125,176,28,122,163,70,99, \quad 0,222,23,166,37$, 207, 101, 59, 202, 136, 150, 234, 104, 254, 78, 11, 231, 211, 138, 117, 63, $144,48,77,158,145,90,164,35,183,184,83,215,181,156,38,233$, $153,10,116,182,213,72,47,223,132,60,140,226,88,69,225,108$, $157,170,237,6,197,85,250,79,134,127,178,167,42,110,198,29$, $169,73,25,103,159,247,218,31,161,102,57,208,155,139,62,81$, $179,20,177,219,253,40,249,194,13,248,131,71,111,152,53,100$, $121,92,187,46,17,201,49,217,124,229,172,236,118,203,98,74$, $245,241,105,97,148,149, \quad 80,228,193,188,173,39,209,252,165,242$

\section{s6:}

$46,145,224,61,59,44,10,12,38,170,23,153,166,245,16,84$, 186, 33, 148, 144, 181, 216, 108, 70, 236, 47, 75, 64, 56, 184, 102, 11, 156, 25, 195, 41, 127, 173, 130, 17, 171, 185, 240, 88, 29, 69, 128, 72, $217,158,83,211,137,229,22,155, \quad 6,21,225,19,135,121,116,207$, 152, 221, 203, 104, 210, 125, 179, 160, 7, 209, 228, 36, 244, 189, 150, 197, 50, 233, 254, 91, 141, 241, 175, 37, 212, 133, 98, 107, 31, 134, 247, 40, 113, 43, 139, 253, 208, 164, 180, 114, 4, 106, 101, 117, 60, 52, 15, 45, $151,26,249,222,82,192,132,39,54,103,214,162,28,177,143,76$, $55,80,35,239,252,157,167,250,193,165,140,146,58,119,24,120$, 66, 8, 105, 109, 51, 219, 169, 92, 0, 201, 110, 30, 213, 94, 174, 97, $67,96,172,123,232,147,168,74,138,218,85,48, \quad 5,223,14,198$, $163,196,86,111,99,237,18,122,230,129,32,176,73,188,200,20$, 136, 63, 53, 142, 90, 79, 77, 205, 27, 1, 95, 149, 9, 93, 100, 42, $71,231,251,131,246,49,183,199,89,238,194,242,215,235,118,204$, $243,57,227,206,115, \quad 3,178,161,78,62,87,13,124,202,191,220$, $159, \quad 2,154,65,248,112,226,255,34,190,68,187,234,81,182,126$

\section{s7:}

$107,180,59,238,174,215,79,126,222,228,145,187,92,7,144,203$, $73,47,138,193,246,183,130,102,156,70,148,57,78,244,134,43$, 159, 209, 216, 219, 188, 205, 46, 63, 160, 44, 32, 140, 253, 168, 15, 95, 139, 68, 223, 234, 106, 117, 94, 118, 231, 241, 176, 236, 81, 20, 60, 157, $54,30,28,178,151,185,245,76,207,86,128,35,251,23,227,116$, $114,198,13,225,25,71,112,190,161,199,85,87,255,213,165,45$, $77,90,124,147,177,220,55,129,53,1,155,194,189,84,169,152$, 
248, 93, 210, 250, 158, 4, 111, 133, 150, 42, 243, 153, 218, 97, 197, 121, 132, 184, 119, 191, 146, 89, 10, 221, 226, 143, 21, 136, 103, 206, 65, 224, 49, 131, 122, 115, 104, 9, 142, 163, 171, 149, 127, 123, 214, 154, 40, 2, 204, 196, 125, 254, 162, 18, 120, 6, 5, 240, 192, 56, 69, 66, 170, 29, $11,58,113,64,26,22,109,201,99,41,82,211,105,27,182,181$, 96, 229, 24, 164, 195, 75, 67, 98, 36, 242, 38, 62, 252, 212, 14, 50, 235, 88, 202, 37, 3, 100, 137, 8, 179, 34, 217, 172, 237, 72, 166, 175, $33,232,230,31,80,108,135,101,48,16,173,186,141,83,239,17$, 19, 51, 249, 247, 167, 52, 39, 91, 200, 233, 110, 74, 12, 208, 61, 0

\section{s8:}

$41,176,88,90,79,174, \quad 72, \quad 10, \quad 6,122,89,185,142,91,131,20$, $54,126,15,205,52,73,158,145,212,236,110,57,213,217,68,181$, $30,133,239,100,25,172,16,252,156,129,180,231,128,248,152,78$, $250,247,207,33,26,144,45,204,55,111,2,228,94,195,138,0$, 48, 22, 251, 136, 121, 77, 139, 115, 92, 160, 201, 171, 155, 101, 103, 170, 186, 242, 64, 235, 215, 56, 83, 99, 187, 42, 23, 150, 40, 183, 234, 157, 206, 203, 216, 148, 229, 3, 179, 13, 125, 21, 226, 5, 31, 132, 230, 7, $241,93,140,193,112,153,249,123,134,1,253,189,65,227,177,199$, $178,86,240,49, \quad 32,75,197,218,50,167,11,39, \quad 59,135,238,141$, 220, 211, 147, 104, 209, 245, 161, 151, 96, 102, 137, 61, 9, 12, 70, 38, $58,106, \quad 35, \quad 53,19,17,210,237,127,120,29,84, \quad 4,24,34,37$, 232, 67, 159, 188, 198, 225, 202, 130, 224, 109, 97, 244, 43, 81, 107, 60, $95,114,219,146,28,105,184,255,47,69,117,143,85,191,119,118$, $36,27,175,173,223,80,124,164,44,182,168,46,196,222,254,149$, $208,71,163,200,165,66,76,243, \quad 8,162,14,98,221,82,51,192$, $246,214,62,63,116,166,233,108,87,74,190,169,113,194,154,18$

\section{s9:}

160, 198, 4, 106, 68, 66, 149, 232, 118, 234, 34, 242, 141, 73, 91, 128, $111,59,254,112,87,161,180,214,213,89,54,147,22,110,179,41$, $101,139,205,182,196,190,210,58, \quad 3,72,229,94,67,116, \quad 52,39$, $76,208,107,244,157,223,119,9,243,99,113,93,148,7,177,150$, $173,195,100,6,250,165,228,114,216,171,145,108, \quad 2,194,226,175$, 83, 206, 219, 40, 17, 86, 55, 236, 32, 156, 146, 97, 21, 122, 251, 70, $8,84, \quad 37,20,16,12,92,239,222,142,143,77,224,129,50,69$, $131,238,199,178,130,144,227,120,162,80,42,240,71,197,245,174$, $33,28,255,103,211,140,18,49,252,136,127,64,126,247,235,217$, $90,166,48,189,105,123,221,57,246,53,172,47,60,14,45,176$, $38,202,104,117,241,62,169,44,193,230,36,121,102,218,79,25$, $13,201,51,135,15,65,186,0,233,74,133,137,155,207,30,43$, 138, 75, 1, 151, 163, 187, 78, 98, 10, 23, 152, 204, 96, 56, 209, 95, 153, 249, 31, 154, 115, 212, 46, 191, 29, 203, 183, 253, 61, 109, 85, 11, $200,26,248,164,134,88,158, \quad 5,24,215,237,231,185,81,27,220$, 


\section{Appendix2:}

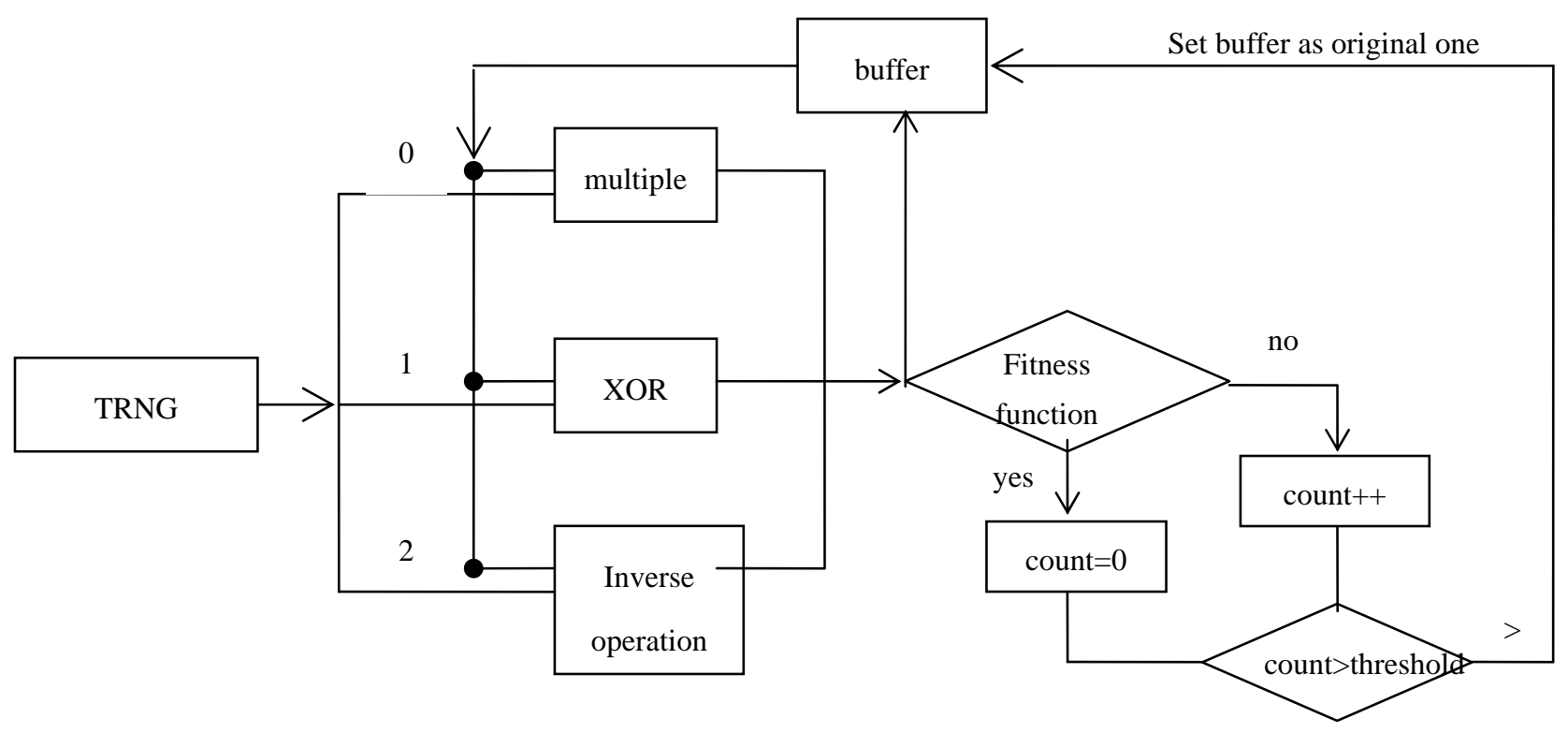

Figure 8: the structure of evolutionary algorithm[meng]

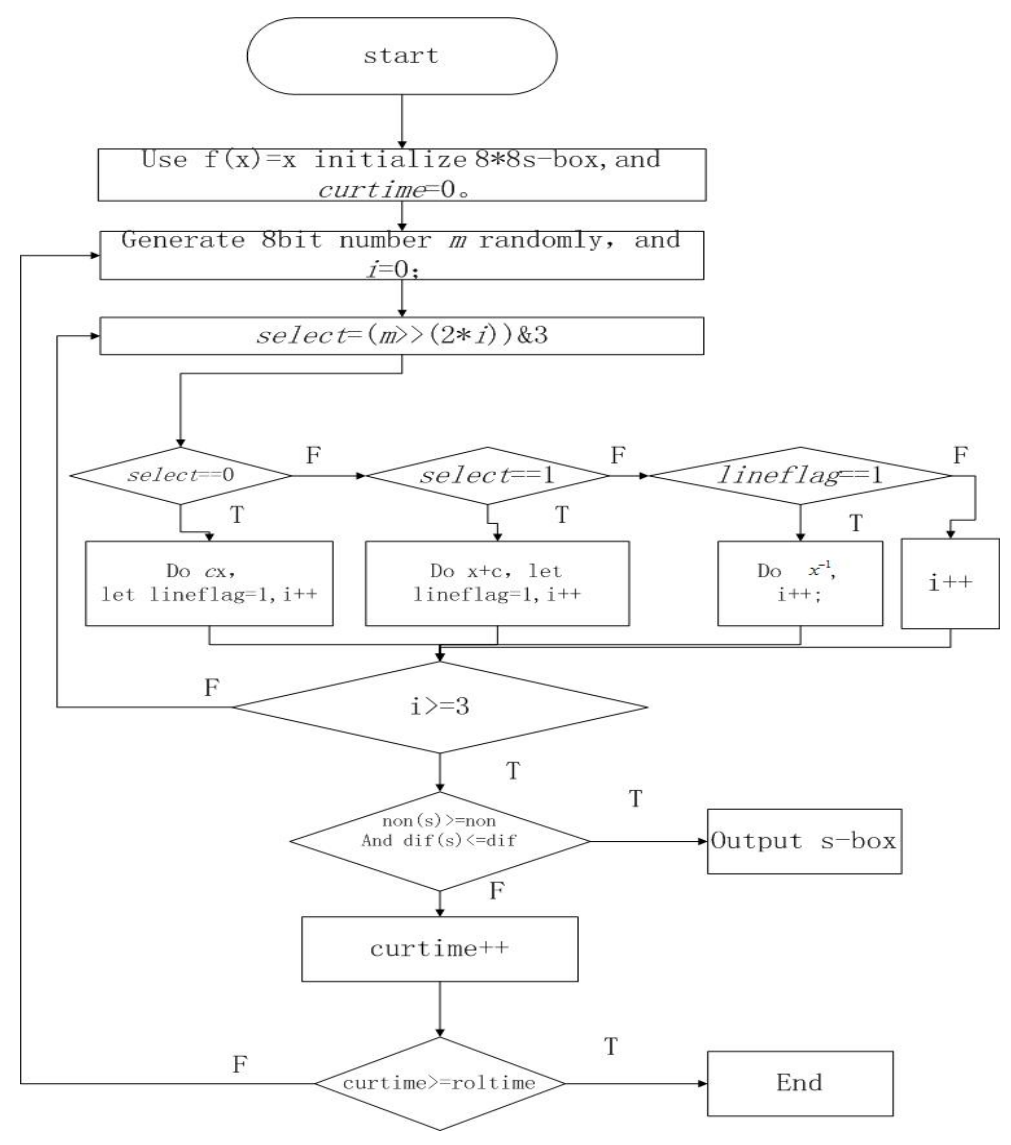


Fugure 9: The flowchart of the algorithm

\section{Reference:}

[1] A. J. Menezes, P. C. Oorschot, S. A. Vanstone. Handbood of applied cryptography(5 ${ }^{\text {th }}$ edition). CRC Press, 2001.

[2] Paul Kocher, Joshua Ja_e, and Benjamin Jun. Differential Power Analysis. Michael Wiener (Ed.): CRYPTO’99, LNCS 1666, pp. 388-397, 1999

[3] E. Biham and A. Shamir, Di_erential Cryptanalysis of the Data Encryption Stan-dard, Springer-Verlag, 1993.

[4] M. Matsui, IThe First Experimental Cryptanalysis of the Data Encryption Standard," Advances in Cryptology: Proceedings of CRYPTO '94, Springer-Verlag, August 1994, pp. 1-11.

[5] http://en.wikipedia.org/wiki/Side-channel_attack

[6] E. Trichina, D.D. Seta, L. Germani. Simplified Adaptive Multiplicative Masking for AES

CHES 2002, LNCS 2523, pp.187-197, 2003.

[7] Tiri K, Hwang D, Hodjat A, et a1. AES—based cryptographic and biometric security coprocessor IC in 0. 18 II m CMOS resistant to side channel power analysis attacks[J]. IEEE Journal of Solid—State Circuits(JSSC), April, 2006, 41(4): 781-792.

[8] Nele Mentens, Benedikt Gierlichs, and Ingrid Verbauwhede, Power and Fault Analysis Resistance in Hardware through Dynamic Reconfiguration, E. Oswald and P. Rohatgi (Eds.): CHES 2008, LNCS 5154, pp. $346-362,2008$.

[9] Yang S, Wolf W, Vijaykrishnan N, et a1. Power attack resistant cryptosystem design: a dynamic voltage and frequency switching approach[c]. Proc. Of Design, Automation and Test in Europe (DATE 2005), Munich, Germany, 2005. 64-69.

[10] Zhang Huan-Guo et al, Research on Evolutionary Cryptosystems and Evolutionary DES, CHINESE JOURNAL OF COMPUTERS 2003 Vol.26 No.12 P.1678-1684.

[11] Min Yang, Qingshu Meng, Huanguo Zhang, Evolutionary Design of Trace Form Bent Function. Available http://eprint.iacr.org. 2005/332.

[12] Qingshu Meng, Min Yang, Huanguo Zhang, Yuzhen Liu, Analysis of Affinely Equivalent Boolean Functions. The First Workshop on Boolean Functions and Application on Cryptography, De Rouen press, France, also available at http://eprint.iacr.org, 2005/025.

[13] Zhangyi Wang, Huanguo Zhang, Qingshu Meng, Differential Cryptanalysis of Hash Functions Based on Evolutionary Computing, ISICA 2007.

[14] Messerges T S, Dabbish E A, Sloan R H. Investigations of power analysis attacks on smartcards[C]. Usenix 
Workshop on Smartcard Technology, Chicago, Illinois, USA, M ay, 1 999, 151-162.

[15] S. Chari, C. Jutla, J. Rao, P. Rohatgi. Towards sound approaches to counteract power-analysis attacks. CRYPTO’99, LNCS 1666,pp398-412,1999.

[16] L. Goubin. A sound method for switching between boolean and arithmetic masking. CHES 2001,LNCS 2162,pp.3-15,2001.

[17] YongBin Zhou, DengGuo Feng. Side-Channel Attacks: Ten Years After Its Publication and the Impacts on Cryptographic Module Security Testing. This paper is invited to appear at the Physical Security Testing Workshop (Hawaii, September 26-29, 2005) held by the National Institute of Standards and Technology (NIST) of USA for discussions on issues specific to physical security testing and security requirements of cryptographic modules.

[18] Pramstaller N, Guerkaynak F K, Haene S, et a1. Towards an AES crypto - chip resistant to differential power analysis[c]. Proc. of the 30th European Solid-State Circuits Conference (ESSCIRC 2004), Leuven, Belgium, 2004.307-310

[19]Akkar M, Giraud C. An implementation of DES and AES.secure against some attacks[c]. Workshop on Cryptographic Hardware and Embedded Systems(Ches 2001). LNCS 21 62. Springer—Verlag, 2001. 309-31 8.

[20]Golic J D, Tymen C. Muhiplicative magking and power analysis of AES[C]. Workshop on Cryptographic Hardware and Embedded Systems (CHES 2002), LNCS 2523, Springer—Verlag. 2002.198—212.

[21] K. Tiri and I. Verbauwhede, A Logic Level Design Methodology for a Secure DPA Resistant ASIC or FPGA Implementation, In Proc. of Design Automation and Test in Europe Conference, pp. 246-251, 2004.

[22] E. Trichina, Combinational Logic Design for AES SubByte Transformation on Masked Data, Cryptology ePrint Archive, 2003/236, 2003.

[23] Tiri K, Verbauwhede I. A digital design flow for secure integrated circuits[J]. IEEE Transactions on Computer Aided Design ofIntegrated Circuits and Systems(TCAD), 2006, 25(7): 1197-1208.

[24] Tiri K, Hwang D, Hodjat A, et a1. AES-based cryptographic

and biometric security coprocessor IC in $0.18 \mathrm{II} \mathrm{m} \mathrm{CMOS} \mathrm{resis-}$

tant to side - channel power analysis attacks[J]. IEEE Journal of

Solid—State Circuits(JSSC), April, 2006, 41(4): 781-792.

[25] Daisuke Suzuki, Minoru Saeki, and Tetsuya Ichikawa. DPA Leakage Models CMOS Logic Circuits. CHES 2005, LNCS 3659, pp. 366-382, 2005

[26] Xilinx. OPB HWICAP,

$\underline{\text { http://www.xilinx.com/bvdocs/ipcenter/data sheet/opb hwicap.pdf }}$

[27] www.altera.com/literature/hb/qts/qts_qii53013.pdf

[28] http://www.xilinx.com/bvdocs/ipcenter/data sheet/Xilinx Power Estimator User Guide.pdf

[29] State intellectural property office of P.R.C, application number: 200910060597.8

[30] C. E. Shannon, A Mathematical Theory of Communication*, Mobile Computing and Communications Review, Volume 5, Number I

[31] Amir Moradi,Mohammad T.Manzuri Shalmani,and Mahmoud Salmasizadeh.A Generalized Method of Differential Fault Attack Against AES Cryptosystem. CHES2006,LNCS4249,pp.91- 100,2006.

[32] http://techcenter.dicder.com/2006/0326/content_150.html 
